
of the LHCb Silicon Tracker

The LHCb experiment

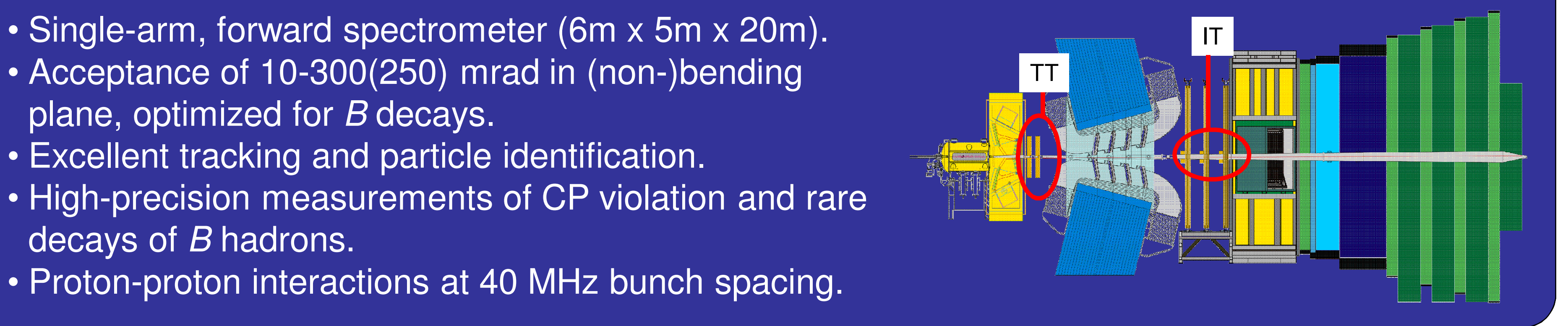

\section{The Silicon Tracker}

- Similar readout electronics with front-end electronics operating at $40 \mathrm{MHz}$.

- Both detectors are operated below $5{ }^{\circ} \mathrm{C}$ to minimize radiation damage.

- Up to 4 (6 inch) sensors bonded together to form longer readout strips (readout sectors).

-Zero-suppression (cluste

$-150 \mathrm{~cm} \times 130 \mathrm{~cm}$ planar station.
- 4 layers (two with $\pm 5^{\circ}$ stereo angle)

- Upstream of dipole magnet

- 64 modules with 14 sensors each.

- Readout of $1,2,3$ and 4 sensor sections.

Strip pitch: $183 \mu \mathrm{m}$

- Sensor thickness: $500 \mu \mathrm{m}$

- Active area: $7.8 \mathrm{~m}^{2}$.

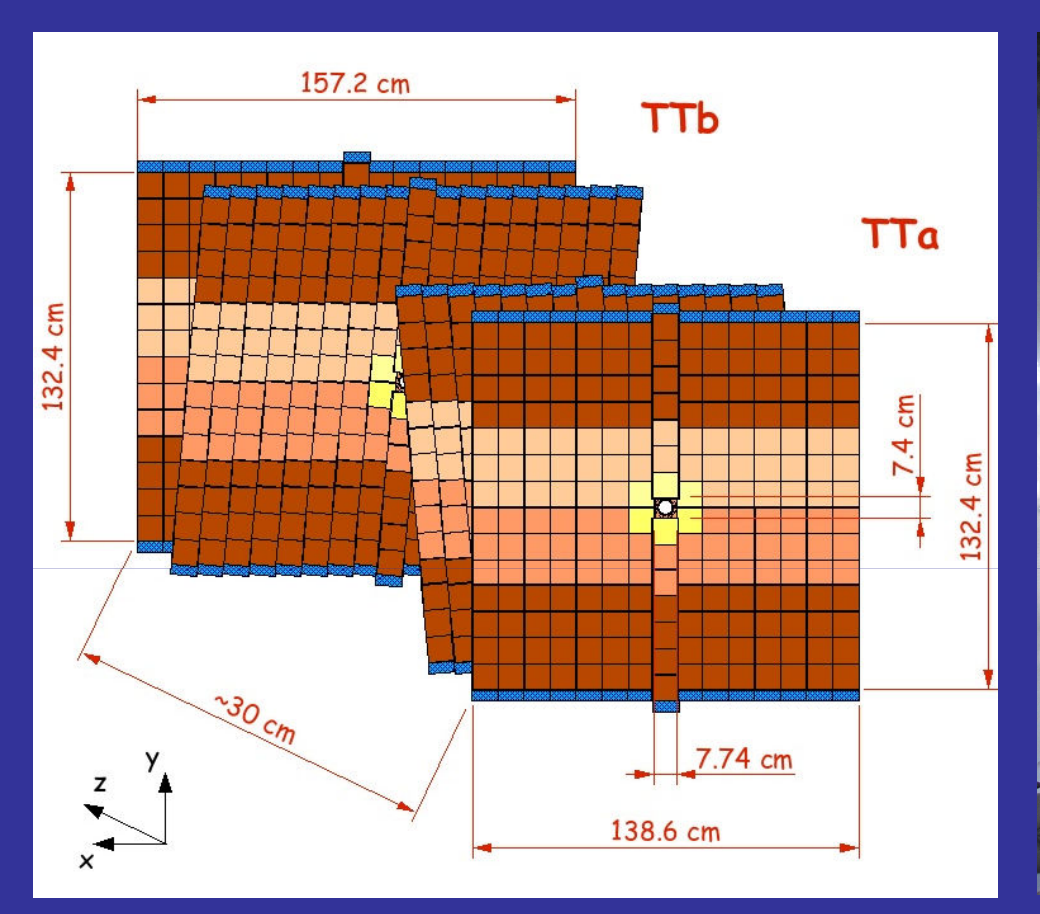

Inner Tracker (IT)

around beam pipe.

- 3 stations with 4 boxes each

- Each box has 4 layers $\left(0^{\circ},+5^{\circ}\right.$

Downstream of dipole magnet

$1 \%$ of acceptance (but $30 \%$ of particles)

Readout of 2-sensor (long) and

-sensor (short) modules.

Strip pitch: $198 \mu \mathrm{m}$

Sensor thickness: $320 \mu \mathrm{m}$ and $410 \mu \mathrm{m}$

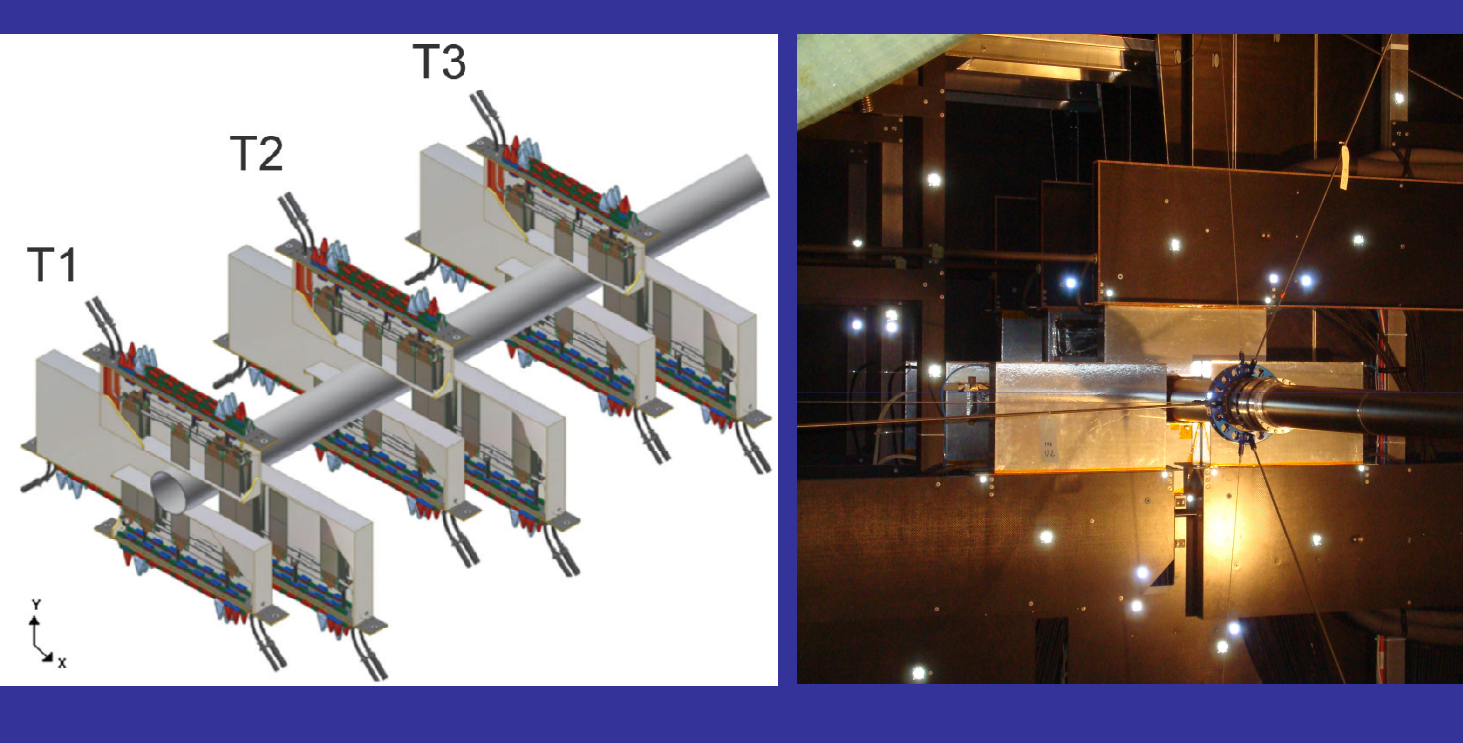

\section{Commissioning}

- Extensive commissioning without beam (comparing noise levels): many readout problems fixed

- Most problem fixed by replacing faulty components (e.g. electronics board, patch panel, cable).

Broken bonds (TT)

- Only innermost bonds broken (staggered bonding).

- 9 readout bonds break on pitch adapter side.

- Many investigations (not reproducible in lab).

- Low loop height innermost bonds is not ideal.

Heel cracks seen on good bonds but mainly on wrong

Material fatique probably induced by stress on wire

ions, thermal cycling

- Number of new broken bonds is decreasing

- No new broken bonds since July 2009.

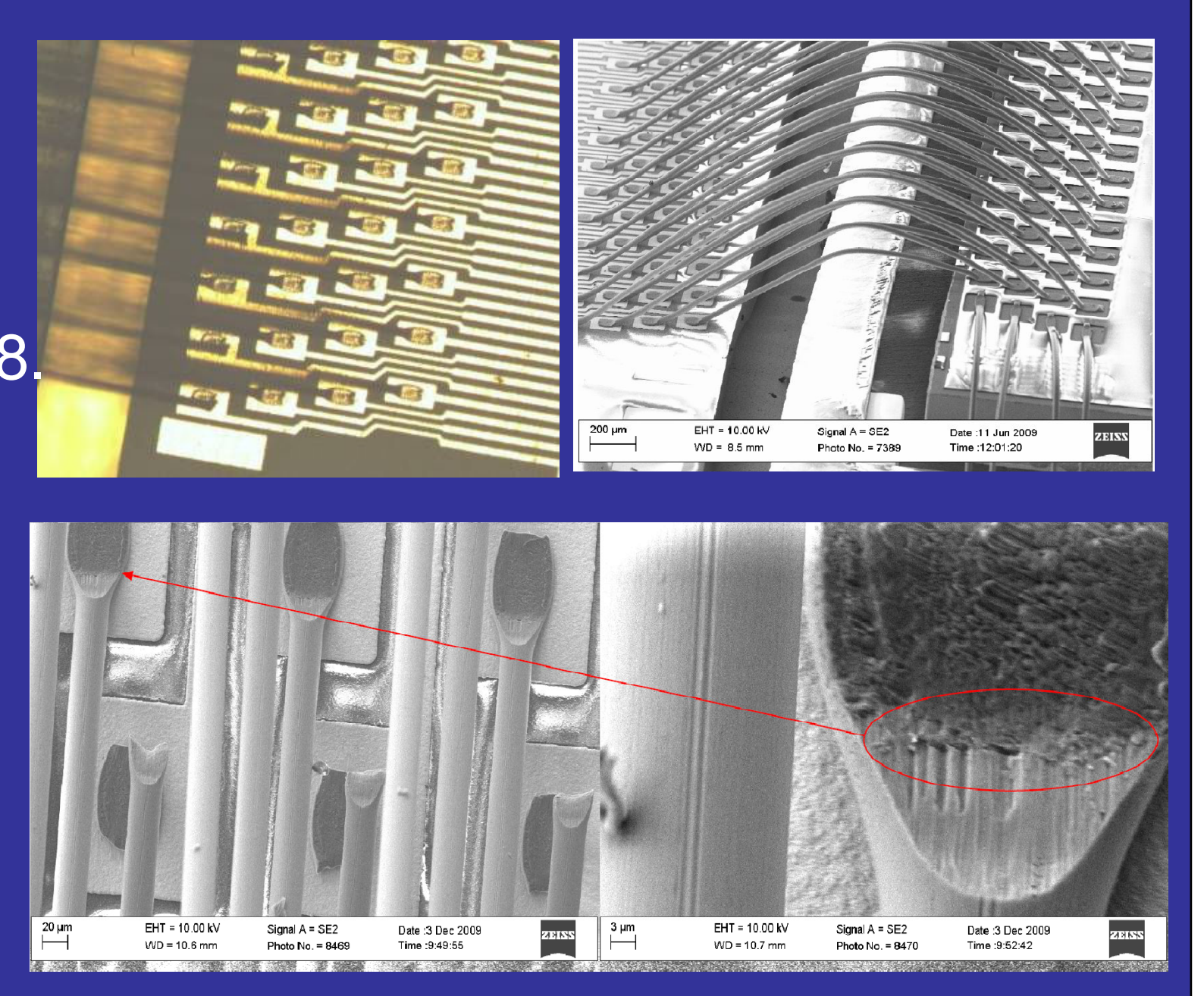

Header cross talk

- FE-chip (Beetle) sends analog data via four output ports.

- Data for each port (32 channels) preceded by

(header bits are encoded as analog signals).

- Header cross talk depends on length of output cable.

- Effect can be corrected for in LHCb readout board (TELL1)

(first two

bits are constant

Correction can be applied on the first 6 strips in each port

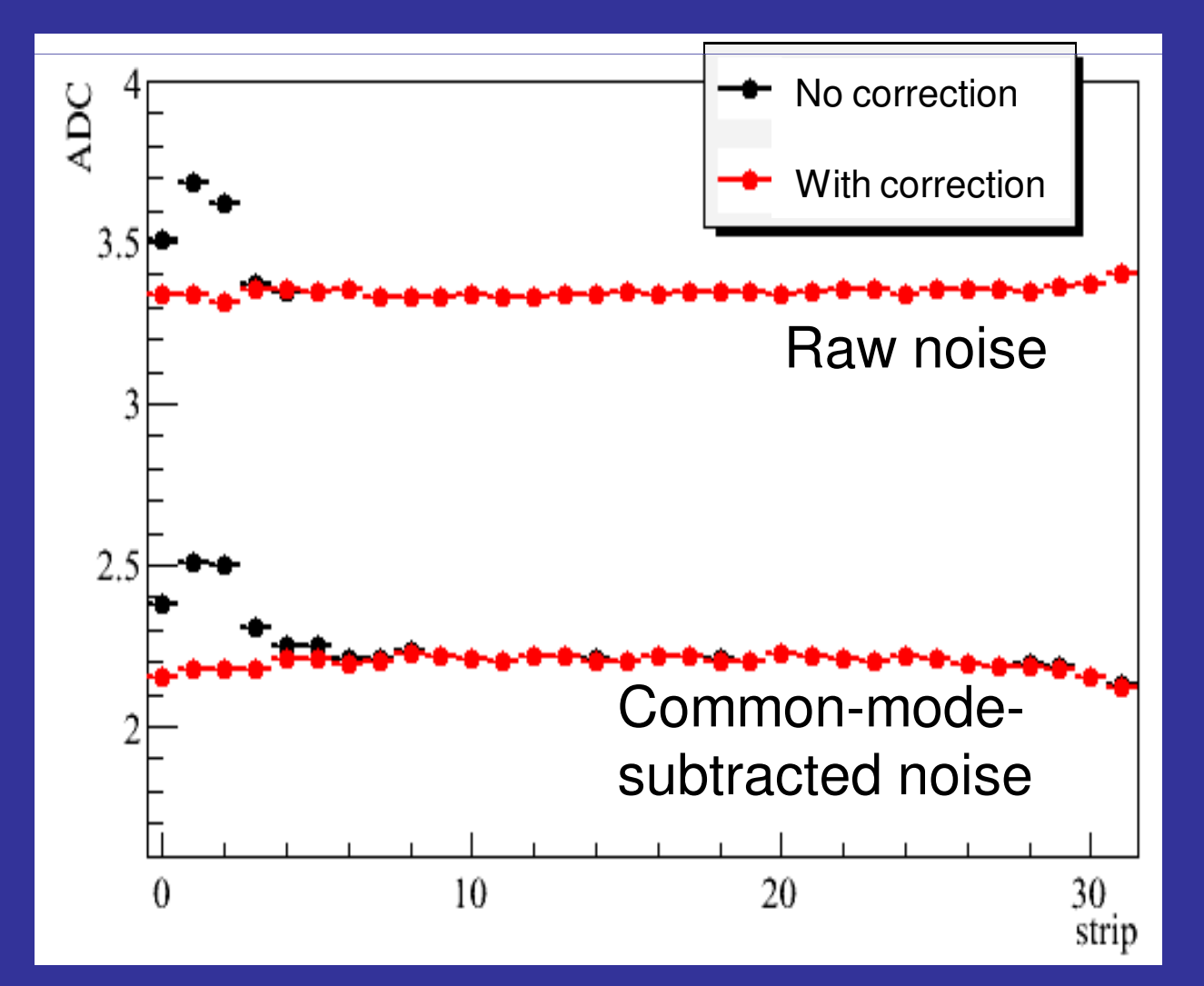

Number of working channels
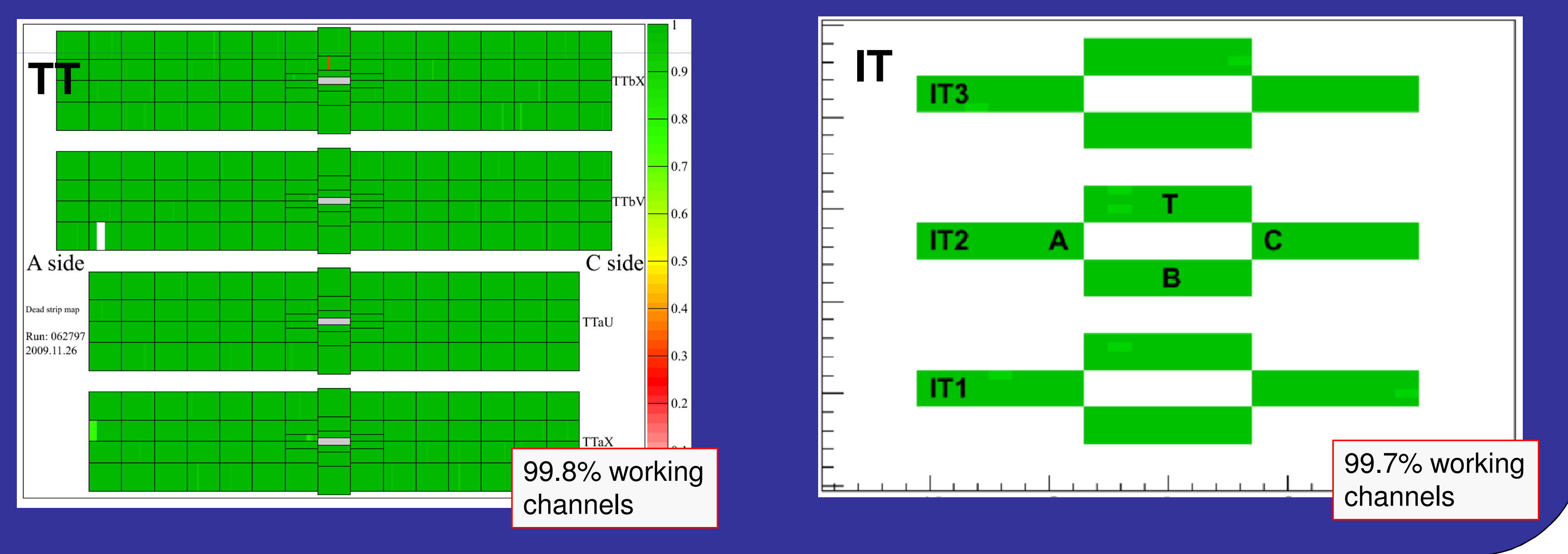

LHC injection tests

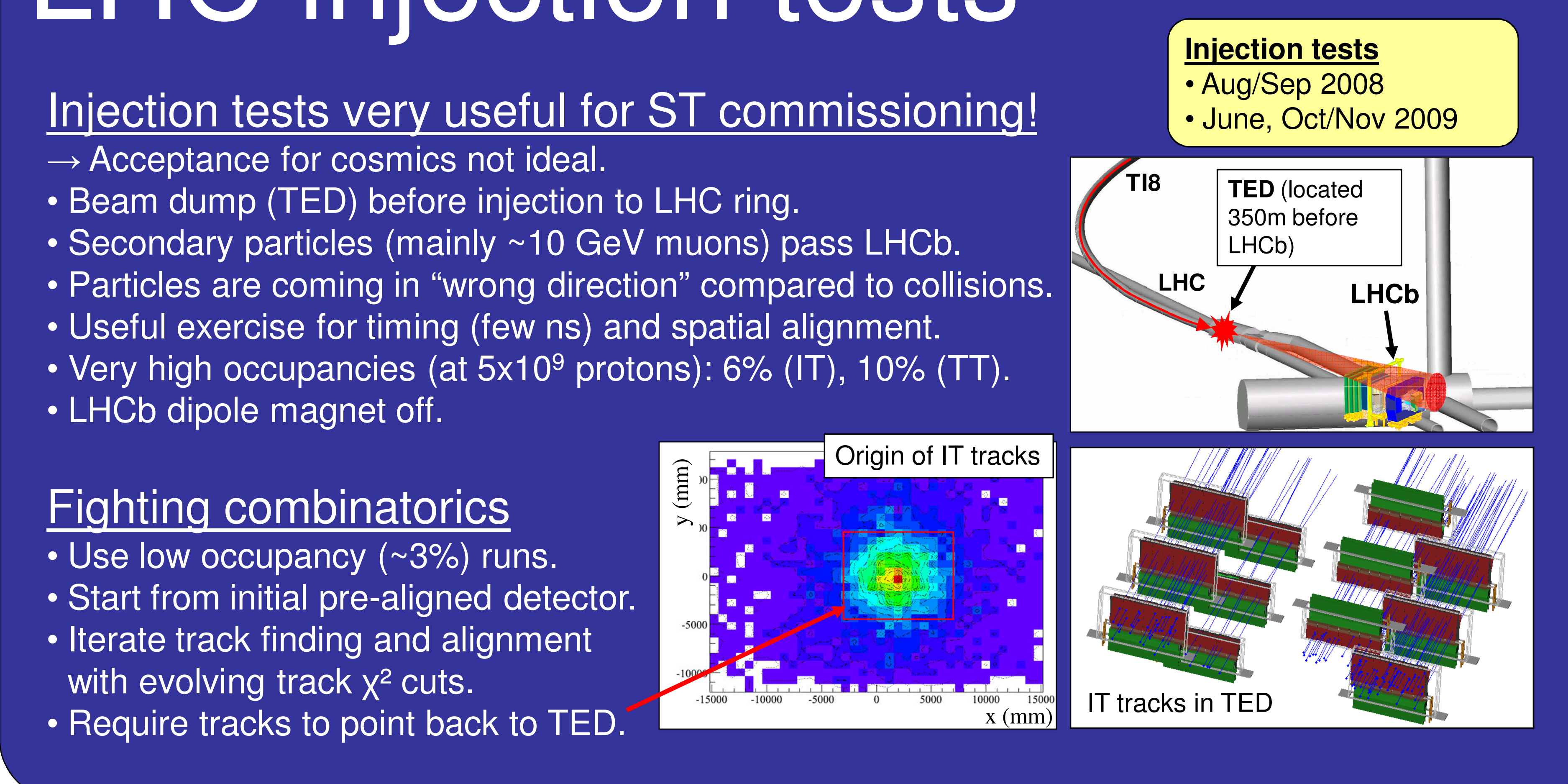

Beam gas \& First collisions

Collected 540k events with all detectors at $450 \mathrm{GeV}$. Highlights from 2009

- Collected 3k events with dipole magnet off.

A wealth of

- First mass peaks at right place.
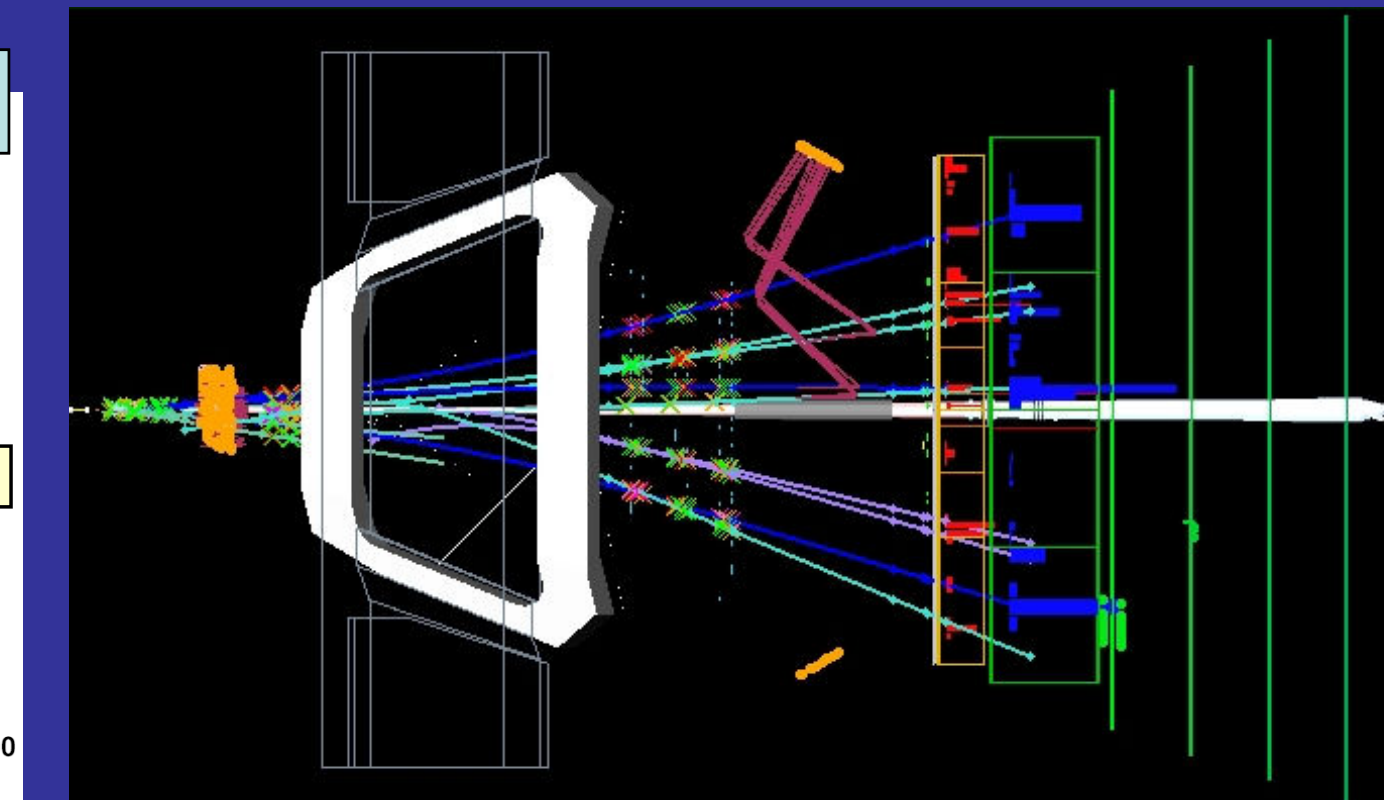

Time alignment

- Initial time alignment done (and practiced) with TED data. Fine-timing (<ns) done with first stable beam (Dec 2009).

- Mear zero-suppressed data $\rightarrow$ not full pulse shape.

Fine-timing will be redone for 2010 run

- Cooling temperature changed from $15^{\circ} \mathrm{C}$ to $5^{\circ} \mathrm{C}$

- FE (Beetle) chip settings changed (slightly higher $\mathrm{S} / \mathrm{N}$ )

Method

- Take non-zero-suppressed data w/ different trigger delays.

- Follow pulse clusters (no bias from capacitive coupling).

- Allows to plot full pulse shape (including undershoot).

- Determine MPV in cluster charge (S/N) distribution.

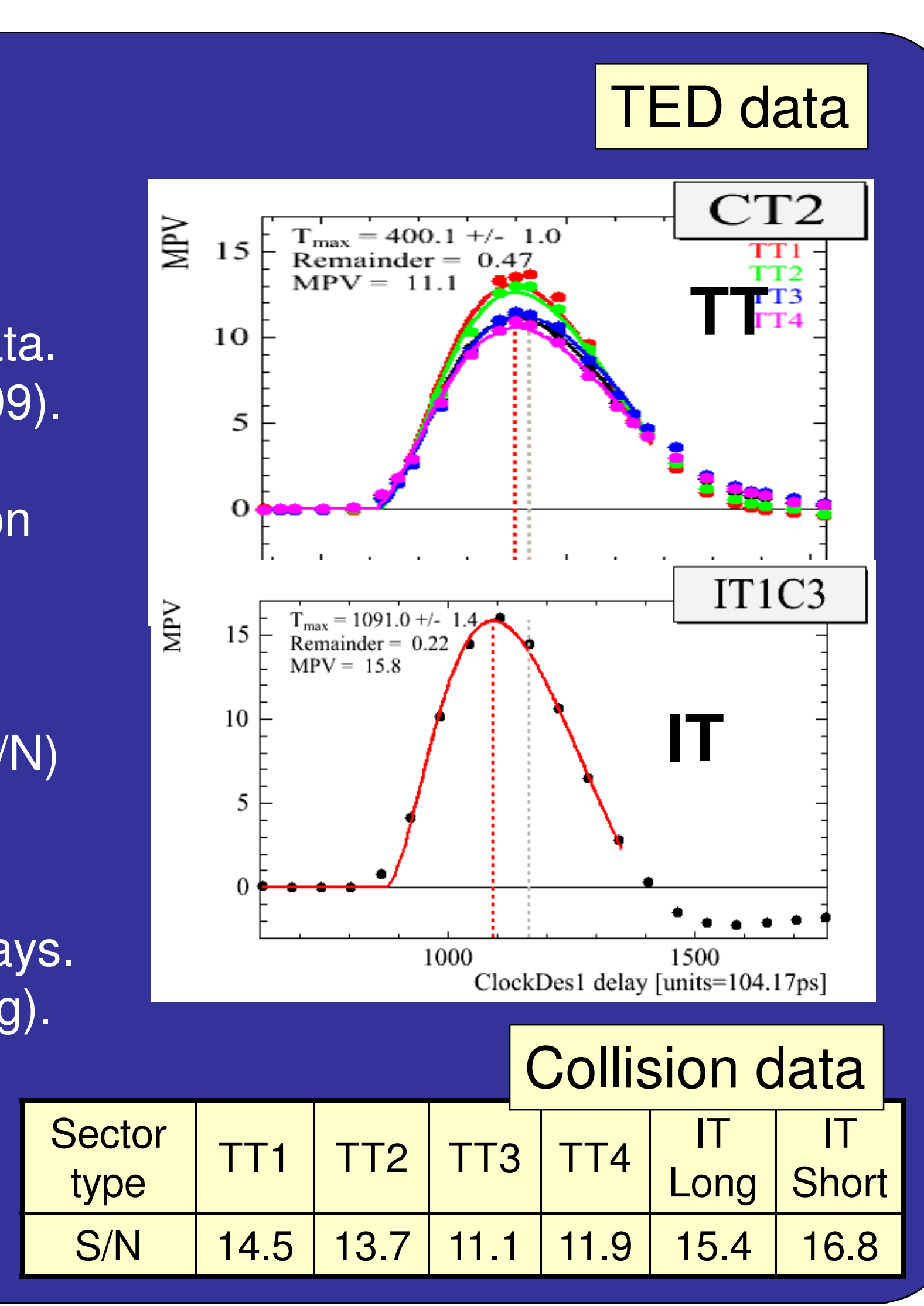

Spatial alignment

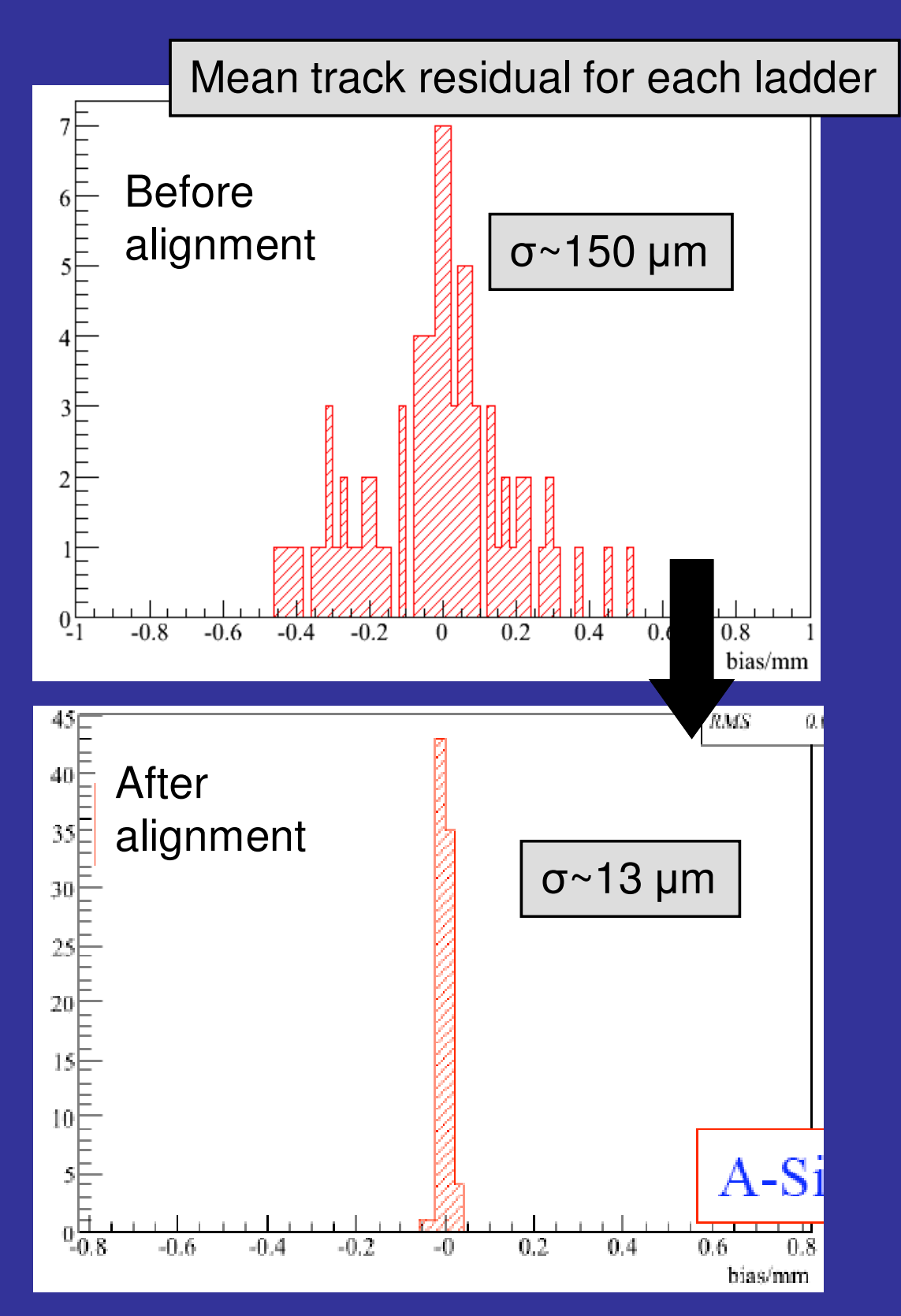

IT alignment with TED data

Use detector survey as starting point.
Use standalone tracking in IT (total $\sim 16 \mathrm{k}$ isolated tracks)

- IT boxes in different positions during TED runs.

- Closed-form alignment with Kalman fitted tracks

- Alignment of boxes (2 translations and 1 rotation), layers

(1 translation and 1 rotation) and ladders (1 translation).

- Ladder alignment precision $\sim 13 \mu \mathrm{m}$.

IT alignment with collision data

- Use TED alignment as starting point

- Use tracks starting from interaction pont

Ladder alignment precision $\sim 15 \mu \mathrm{m}$.

- Unbiased residuals indicate additional residual misalignment.

- E.g. still working on alignment between boxes using overlaps.

TT alignment

Only 4 layers $\rightarrow$ nc

- TED data only used for aligning of full TT station in 2 do

- Beam collision data used for first module alignment with

tracks starting from interaction point.

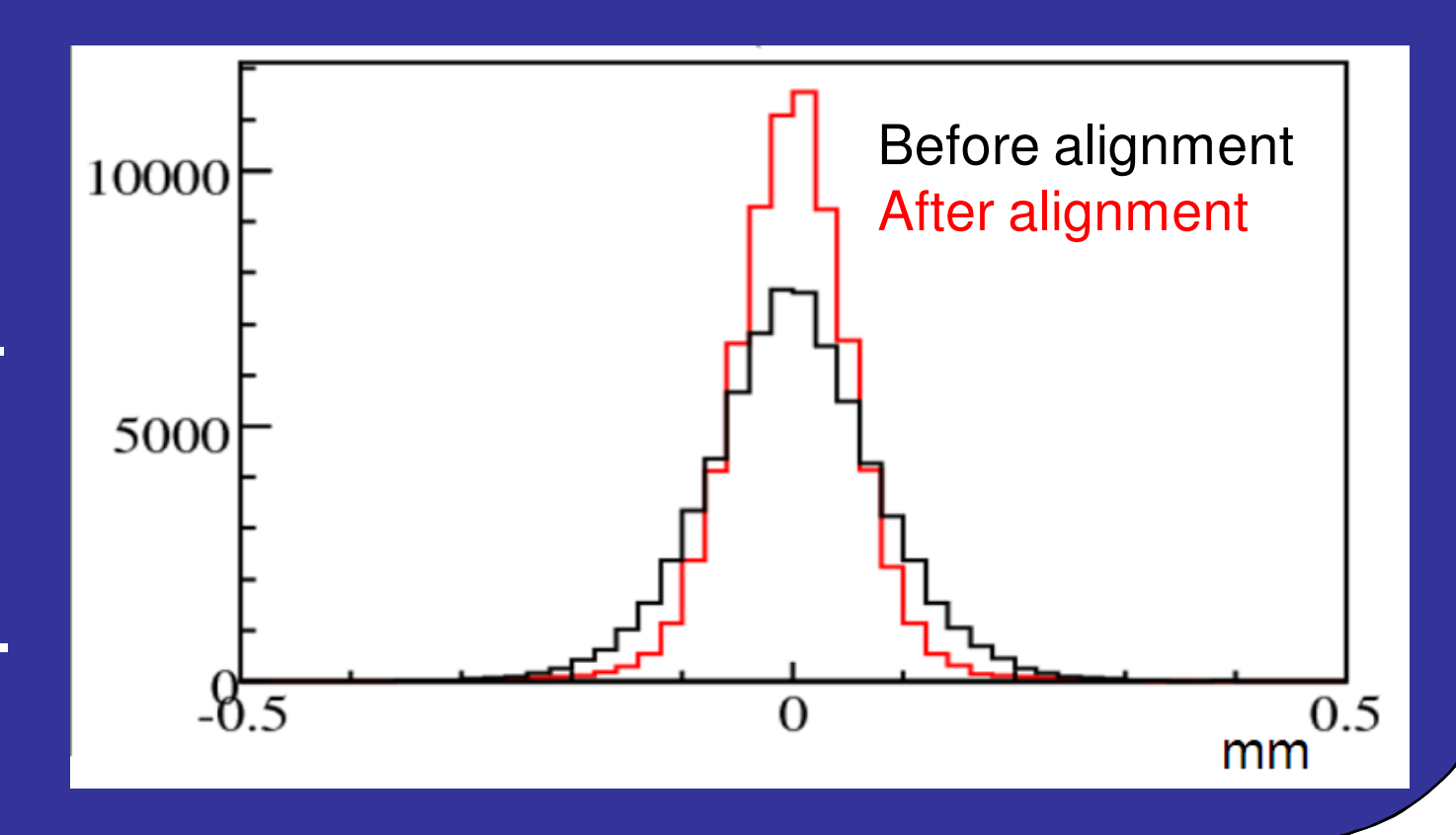

